\title{
Netzplanung und -betrieb
}

R. Schürhuber

angenommen am 3. November 2021, online publiziert am 16. November 2021

(c) Springer-Verlag GmbH Austria, ein Teil von Springer Nature 2021

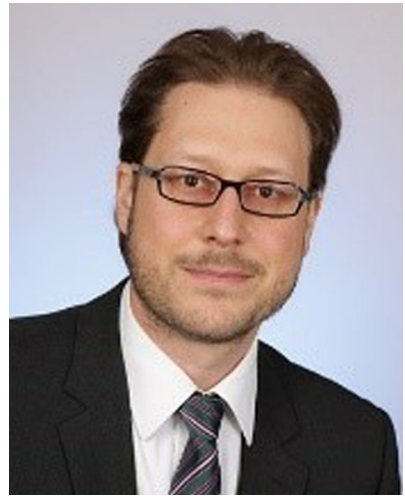

Univ.-Prof. DDipl.-Ing.

Dr.techn. Schürhuber, Robert
Liebe Leser/innen!

Es freut mich sehr, die Gelegenheit bekommen zu haben, eine Ausgabe der Zeitschrift e\&i zu gestalten! Wie viele von Ihnen wissen, sind im deutschsprachigen Raum Zeitschriften mit Originalpublikationen sowie einem Überblick über das Geschehen in der Branche, welches nicht primär marketingorientiert ist, rar gesät. Umso höher ist daher das Engagement des Österreichischen Verbands für Elektrotechnik zu bewerten, sich in dieser Richtung zu engagieren, da es abgesehen von der Wissensvermittlung auch fördernd für die Zusammenarbeit und den Austausch von Neuigkeiten in der Branche und zwischen den Forschungseinrichtungen wirkt.

Im Bereich der Energietechnik stehen wir aktuell wohl vor den größten Herausforderungen seit dem Aufbau des Systems. Der Klimawandel und die damit verbundene bindungslose und unmittelbar umzusetzende Umstrukturierung des Energiesystems und der Energiewirtschaft auf eine klimaneutrale Betriebsweise bedingen Veränderung im gesamten System. Handlungsbedarf besteht also nicht nur in einzelnen Segmenten der Energietechnik, sondern in der ganzen Kette von Erzeugung, Transport und Verbrauch. Bewusst verwende ich hier nicht den Begriff "elektrische Energietechnik", denn die Umstellung der gesamten Energiewirtschaft beinhaltet alle Sektoren, was sich auch an vielfältigen Aktivitäten in Bezug auf die Sektorkopplung zeigt. Diese Verbindung bislang meist getrennter Energieströme entspricht vom technischen her in vielen Bereichen einem Ausbau der Elektrifizierung. Wenn wir bedenken, dass unser Gesamtenergieverbrauch aktuell nur zu rund einem Viertel elektrischer Natur ist, wird sofort klar, dass besonders der elektrische Sektor mit gewaltigen Herausforderungen konfrontiert ist, welche es zu meistern gilt.
Diese vielen neuen Aufgaben spiegeln sich auch in der großen Anzahl eingereichter Originalarbeiten wider, aufgrund derer die Arbeiten auf zwei Ausgaben der Zeitschrift aufgeteilt werden mussten. In dieser Ausgabe liegt der Schwerpunkt auf systemischen und netztechnischen Betrachtungen, während sich die nächste Ausgabe mehr auf die Betriebsmittel konzentriert.

Natürlich wird hier nur ein grober Ausschnitt aus der aktuellen Forschung dargestellt, aber anhand der Artikel sind deutlich zwei der ganz großen Themenkreise zu sehen: der Umgang mit einer stets größer werdenden Anlagenvariabilität, sowohl im Bereich der Erzeugung als auch im Bereich des Verbrauchs, sowie eine Forderung nach Flexibilität im Netzbetrieb auf allen Netzebenen. Der erste Aspekt zeigt sich beispielsweise darin, welche Bedeutung Themen zum Netzanschluss in den letzten Jahren gewonnen haben. Der zweite Aspekt findet sich $u$. a. in der Untersuchung der Anwendung von Optimierungsmethoden bis zum Hausanschluss und das in einer Perfektion, welche vor nicht langer Zeit hauptsächlich im Bereich des Übertragungsnetzes üblich war.

Aber es ist nicht zu vergessen, dass trotz neuer Herausforderungen die Physik der Erzeugung, Verteilung und Übertragung nach wie vor durch die Maxwellschen Gleichungen bestimmt ist. Die dadurch definierten Randbedingungen bleiben gleich und können durch keine noch so raffinierte Optimierung übergangen werden. Die klassischen elektrotechnischen Aspekte dürfen damit nicht aus den Augen verloren werden und stellen uns oftmals vor größere Herausforderungen denn je, wie z. B. im Artikel über den zukünftigen Wärmepumpenzuwachs nachzulesen ist.

Insgesamt halten Sie mit dieser Ausgabe einen interessanten Einblick über die vielfältigen aktuellen Entwicklungen in Händen, und ich wünsche Ihnen ein interessantes Schmökern darin!

Mit besten Grüßen aus Graz

Prof. Robert Schürhuber

Hinweis des Verlags Der Verlag bleibt in Hinblick auf geografische Zuordnungen und Gebietsbezeichnungen in veröffentlichten Karten und Institutsadressen neutral.

Schürhuber, Robert, Institut für Elektrische Anlagen und Netze, Technische Universität Graz, Inffeldgasse 18//, 8010 Graz, Österreich (E-Mail: robert.schuerhuber@tugraz.at) 